\title{
CONTENT RECARVING AS SUBJECT MATTER RESTRICTION
}

\author{
VINCENZO CICCARELLI \\ https://orcid.org/0000-0002-8996-9170 \\ University of Campinas \\ Department of Philosophy \\ Rua Cora Coralina, 100, Cidade Universitária \\ Campinas, SP, 13083-869 \\ Brazil \\ ciccarelli.vin@gmail.com
}

\begin{abstract}
Article info
CDD: 193

Received: 14.05.2018; Revised: 12.12.2018; Accepted: 02.01.2019

DOI: http://dx.doi.org/10.1590/0100-6045.2019.V42N1.VC
\end{abstract}

\section{Keywords}

Abstraction principles

Content recarving

Subject matter

\begin{abstract}
In this artide I offer an explicating interpretation of the procedure of content recarving as described by Frege in $\$ 64$ of the Foundations of Arithmetic. I argue that the procedure of content recarving may be interpreted as an operation that while restricting the subject matter of a sentence, performs a generalization on what the sentence says about its subject matter. The characterization of the recarving operation is given in the setting of Yablo's theory of subject matter and it is based on the relation of determination between properties. The main advantage of the proposal is its generality, for it is applicable not just to the case of abstraction principles.
\end{abstract}




\section{INTRODUCTION}

In section 64 of the Foundations of Arithmetic (Frege, 1950), Frege writes:

The judgment 'line $a$ is parallel to line $b$ ' [...] may be taken as an identity. If we do this, we obtain the concept of direction and say: 'the direction of line $a$ is identical with the direction of line $b$. Thus we replace the symbol || by the more generic symbol =, through removing what is specific in the content of the former and dividing it between $a$ and $b$. We carve up the content in a way different from the original way and this yields us a new concept.

This passage is of no secondary importance: it may be read as the justification for the fact that a statement asserting that a certain equivalence relation holds may be taken as an identity. A family of definitions may be seen as logically grounded in the procedure described in the quoted passage; using a terminology that probably Frege would not have endorsed, they have been labeled abstraction principles. One of the most interesting cases of abstraction principle is the implicit definition of the concept of cardinal number, also known as Hume's Principle; it states that the sentence 'there is a one-to-one correspondence between all instances of the concept $F$ and those of the concept $G$ ' may be taken as the identity 'the cardinal number of the concept $F$ is identical to the cardinal number of the concept $G$. To highlight the similarity between the case of cardinal numbers and that of directions, note that the open sentence 'there is a one-toone correspondence between all instances of the concept $X$ and those of the concept $Y^{\prime}$ defines a higher-order 
equivalence relation that, for sake of brevity, we will call equinumerousity.

It has to be admitted: the passage in $\$ 64$ is partially obscure. It is unclear what should be taken as the content of the symbol 'Il '; moreover, it is even less clear how to intend the operation of removing what is specific in the content of this symbol and dividing it between $a$ and $b$.

The first step toward a satisfactory interpretation of the quoted passage is to make explicit the aspects of Frege's procedure that are immediately understandable. Frege is implying that there may be different ways of carving up the same content, i.e. that the same content may be "organized" in different ways. Moreover, Frege is implying a special relation between (all?) carvings of the same content: the relation that holds whenever one carving is obtained by performing a certain re-conceptualization of another. The operation of removing part of the content of the relation of parallelism and distributing it between its relata may be seen as an example of the operation that converts a carving into another. I call this kind of operation content recarving. It is worth emphasizing that content recarving has been defined as a class of operations, i.e. the class including all operations that convert a carving into another; thus I am open to the possibility of there being procedures of recarving a content different from that described by Frege. Note that Frege is by no means implying that all carvings of the same content must be one the recarving of the other ${ }^{1}$.

According to these remarks, a satisfactory analysis of Frege's procedure must make sense of the notion of content, of the idea that the same content may be carved

1 The view that two sentences may be different carvings of the same content while not being one the recarving of the other is defended Yablo (2014) while considered as "incomprehensible" by Potter and Smiley (2001). 
up in different ways, and provide a general account of the operation of content recarving. This paper aims at proposing such an analysis; my proposal consists in defining the content of a sentence $\varphi$ as the proposition expressed by $\varphi$, the carving of a content as the specification of the exact subject matter of a certain sentence, and the operation of content recarving as subject matter restriction, where this restriction must be defined according to specific criteria. Roughly speaking, the fundamental idea is that given a sentence $\varphi$ and its subject matter $m$, the operation of content recarving consists in restricting the attention to the aspect of $m$ which is the most relevant to 'what $\varphi$ says about $m$.

The semantic setting is the semantics of possible worlds, while the theoretical context is the account of the notion of subject matter given by Lewis (1988) and improved by Yablo (2014). The main theoretical advantages of the proposal are:

1) a general theory of content recarving that does not apply just to abstraction principles;

2) an explication of Frege's idea that, by going far beyond the level of interpretation, allows for the evaluation of the philosophical consequences of the proposal in a context wider than that of Frege's logicist project.

In section $2 \mathrm{I}$ will give a brief exposition of the theory of subject matter as proposed by Lewis and improved by Yablo; in section $3 \mathrm{I}$ will give a rigorous definition of 'carving up a content'; in section $4 \mathrm{I}$ attempt a first definition of 'content recarving' and I will present the main philosophical difficulty associated with this definition; in section 5 I will improve the definition of section 4 and in section 6 I will give some conclusive remarks. 


\section{LEWIS AND YABLO ON SUBJECT MATTER}

The most immediate account of the subject matter of a given sentence is given by Ryle (1933): a sentence $S$ is about $\mathrm{m}$ iff $\mathrm{S}$ mentions $\mathrm{m}$. However, this account suffers from several limitations: for instance, the sentence 'Brazil has the largest tropical forest' is about Brazil, but also about South America, even though South America is not explicitly mentioned. An improvement of Ryle's proposal is Goodman's account (Goodman, 1961): a sentence S is about $\mathrm{m}$ iff either $\mathrm{m}$ is mentioned by $\mathrm{S}$ or by one of $\mathrm{S}$ 's consequences. If Ryle's account is too narrow, Goodman's improvement seems to be too wide: the sentence 'Brazil has the largest tropical forest or the Congress of Vienna started in 1814' is about the Congress of Vienna and is a consequence of 'Brazil has the largest tropical forest': thus 'Brazil has the largest tropical forest' is about the Congress of Vienna, which is evidently false.

In (Lewis, 1988) a purely semantic account of subject matter is given: a subject matter is presented as some kind of "part" of a possible world. Two worlds $w_{1}$ and $w_{2}$ having a certain part $p$ in common are indistinguishable whenever $p$ is concerned, i.e. a sentence that is entirely about $p$ cannot have different truth-values in $\mathrm{w}_{1}$ and $\mathrm{w}_{2}{ }^{2}$. This remark leads Lewis to make a further step: two worlds that are indistinguishable with respect to a certain part $p$, stand in a certain equivalence relation associated with $p$; call this relation $\equiv_{p}$; the introduction of this relation allows for the definition of a criterion for saying whether a certain

\footnotetext{
2 For sake of simplicity I speak of parts in common between possible worlds. This does not imply that the theory of subject matter presupposes world overlapping; the reader who thinks of world overlapping as untenable may replace 'w1 and w2 have a part in common' with 'there is a part of w1 that is an intrinsic duplicate of a part of w2'.
}

Manuscrito-Rev. Int. Fil. Campinas, v. 42, n. 1, pp. 45-90, Jan-Mar. 2019. 
sentence $S$ is entirely about a certain world part $p$ or not: $S$ is about $p$ iff for every two worlds $w_{1}, w_{2}$, if $w_{1} \equiv_{p} w_{2}$, then the truth-value of $S$ in $w_{1}$ is identical to the truth-value of $S$ in $w_{2}$.

This account of subject matter seems to be promising; however, as Yablo noted, it works only with parts-based subject matters. For instance, it is easy to see that the sentence 'The congress of Vienna started in 1814' as a sentence about 1814, and it also is easy to see that 1814 is a temporal part of a possible world. Similarly, the sentence 'Brazil has the largest tropical forest' is about Brazil, and there are no particular difficulties in considering Brazil as part of a possible world. Yet matters stand in a more complicate way with other examples: the sentence 'There are $n$ stars' is supposedly about the number of stars in the universe and to say that the number of stars is a part of a possible world seems to be understandable only at the cost of substantially altering the meaning of the word 'part'. As Yablo remarks, "Astronomers have not discovered a "starcounter" part of the universe, such that worlds agree in how many stars they contain if and only if the one's counter is an intrinsic duplicate of the other's " ${ }^{3}$. In other words, the number of stars is not a parts-based subject matter. Yablo's solution to the difficulty is to generalize the notion of parts-based subject matter by introducing the notion of partitions-based subject matter. What does it mean to say that a subject-matter is partitions-based? Consider the case of the number of stars; surely we have no guarantees that there is a part of possible worlds that we may point at and say that it is the number of stars. However, it is still possible to define an equivalence relation between worlds agreeing on how many stars there are. Let $\equiv_{n o s}$ be this relation; we may apply Lew is' criterion and say: a sentence $S$ is wholly about the number of stars iff for any

${ }^{3}$ (Yablo, 2014), p. 25

Manuscrito-Rev. Int. Fil. Campinas, v. 42, n. 1, pp. 45-90, Jan-Mar. 2019. 
two worlds $w_{1}, w_{2}$, if $w_{1} \equiv_{n o s} w_{2}$, then the truth-value of $S$ in $w_{1}$ is identical to the truth-value of $S$ in $w_{2}$.

Given that the number of stars seems to be a subject matter, what kind of entity is it qua subject matter? It cannot be a physical part of a world. At this point the shift from the notion of part to that of partition is crucial, for it allows for the needed generalization: the equivalence relation $\equiv_{n o s}$ divides the logical space into cells, i.e. it partitions the logical space, where each cell is composed of all possible worlds agreeing on how many stars there are. Thus we may identify the subject matter number of stars with the partitioning of the logical space defined by the equivalence relation $\equiv_{n o s}$. Clearly, if this generalization works well enough from a logical point view, it introduces an important distinction at the ontological level: when the theory was concerned only with parts-based subject matters we had no problem in identifying a certain subject matter with a certain entity existing in some possible worlds; nonetheless, when we introduce the notion of partitionsbased subject matters, an ontological distinction is needed, for now the subject matter 1814 , i.e. the partition of the logical space including all worlds agreeing on what happened in 1814, no more coincides with the temporal part 1814.

The theoretical move of generalizing the notion of part by introducing that of partition has the significant advantage of providing for all cases: whenever a way of partitioning the logical space is given according to a partial indistinguishability between worlds, a subject matter also is given. And given a subject matter $m$, Lewis' criterion for saying whether a certain sentence $S$ is wholly about $m$ is immediately applicable: if worlds belonging to the same $\mathrm{m}$ cell assign to $S$ the same truth-value, then $S$ is about $m$, otherwise it is not. In other words, $S$ is about $m$ iff the set of possible worlds in which $S$ is true (false) is partitioned by $m$. 
The theory of partitions-based subject matters has another significant advantage: it allows for the definition of interesting relations between subject matters. For instance, it allows for the definition of the relation of inclusion of one subject matter into another, or, in different terms, it allows to say when a subject matter is smaller than another. Consider the case of 1814 as a subject matter: worlds agreeing on what happened in 1814 belong to the same cell. Intuitively, the subject matter XIX century includes the subject matter 1814, for the amount of information about the events occurred in the XIX century includes the information about the events that occurred on 1814; this intuition is confirmed by the theory. Indeed, worlds agreeing on what happened in the XIX century must agree on what happened on 1814 but the contrary does not hold. As a consequence, each 1814-partition must include a XIX century-partition as proper subset; generalizing this result we may say that the wider the subject matter, the finer the partitioning of the logical space. This conclusion is easily explainable: the wider the subject matter, the bigger the amount of information it conveys; the bigger the amount of conveyed information, the harder the agreement between two possible worlds; the harder the agreement between possible worlds, the finer the partitioning of the logical space.

The relation of 'larger than' or 'smaller than' applied to subject matters allows us to determine two extreme cases. Consider a sentence $S$; I will refer by the expression 'Spartitioning' to the way the sentence $S$ partitions the logical space, i.e. the set of all possible worlds in which $S$ is true and the set of possible worlds in which $S$ is false. An extreme case is represented by the coarser subpartitioning of the $S$-partitioning, i.e. the $S$-partitioning itself (an improper subpartitioning). Call mins this subject matter: worlds agree on mins iff they agree on the truth-value of $S$. In other words, the subject matter mins is nothing but 'the 
truth-value of $S$ ' or 'whether $S$ is true or not'. Clearly $S$ is about 'whether $S$ is true or not', yet at the same time it seems to say something more. This because mins is the smallest subject matter on which $S$ is about. Another extreme case is the one in which as subpartitioning of the $S$-partitioning we consider the one that divides the logical space into singleton cells. In this case no two worlds belong to the same cell, i.e. the subject matter is such that it is impossible for two worlds to agree on it. We may characterize this subject matter as 'how the world is': it is the largest subject matter. Clearly, $S$ is about how the world is'; yet at the same time it seems to say something less. The notion underlying these considerations is that of exact subject matter: $S$ is exactly about $m$ iff $S$ is wholly concerned with $m$ and it doesn't care about anything else.

We may say that the sentence $S$ is exactly about a certain subject matter that lies in between the largest one and smallest one. For simplicity of exposition, I say that a sentence $\varphi$ is genuinely about $m$ iff $m$ strictly lies between the largest and the smallest subject matter. The exact subject matter of a sentence $\varphi$ is one of the genuine subject matters on which $\varphi$ is about.

Yablo introduces the notion of ways of being true (false) to define the grade of fineness of the subpartitioning associated with the exact subject matter of a certain sentence. "Sentential subject matter should be at most as fine-grained as ways of being true" 4 - he writes. To clarify the notion of ways of being true, it may help to refer to one of Yablo's examples; consider the sentence:

The U.S. president in 2001 is a senator's son. $(\varphi)$

${ }^{4}($ Yablo, 2014), p.41

Manuscrito-Rev. Int. Fil. Campinas, v. 42, n. 1, pp. 45-90, Jan-Mar. 2019. 
In the actual world $w_{a}$ the U.S. president in 2001 was Dubya, son of former senator George H.W. Bush; suppose that in a possible world $w^{*}$ different from $w_{a}$, the U.S. president in 2001 was $\mathrm{Al}$ Gore, son of the former senator Albert Gore Sr. Both $w_{a}$ and $w^{*}$ make the sentence $\varphi$ true, however there is a substantial difference between the ways in which $\varphi$ obtains in the two worlds: there is a change in personnel, i.e. the U.S. president and his father are different persons. Let $s$ be the subject matter of $\varphi$; Yablo w rites:

This is enough, it seems, to change the state of things where $s$ is concerned. A transworld reporter on the $\varphi$ beat could not plausibly claim that there was nothing to report - that it doesn't matter, from a subject matter of $\varphi$ perspective, who plays the two key roles. A change in personnel is more newsworthy than a change in the price of cotton. It is pretty clear why. The personnel change is a change in the individuals witnessing $\varphi$ 's truth; a change in the witnesses affects how $\varphi$ is true; and changes in how a sentence is true cannot be changes in an aspect of reality that $\varphi$ is not even about.

My understanding of the passage is that ways of being true (false) of a sentence $\varphi$ are univocally determined by the collection of the verifiers of $\varphi$, where by the word 'verifier of $\varphi$ ' I mean 'what witnesses the truth of $\varphi$ '; a sentence cannot be true (false) in the same way in two different worlds in which is verified (falsified) by different entities. According to this preliminary consideration, we may identify the ways of being true or false of a sentence $S$ with the part of the world that is determinant for the assignment of the truth-value to $S$. Or, in different words, the part of a possible world that witnesses the truth-value 
of the sentence. For instance, the truth-value of 'Socrates is wise' is determined by the part of world corresponding to the individual Socrates; the truth-value of 'There are no zombies' is determined by the extension of the property ' $x$ is a zombie' in a certain world: if this extension is empty than it corresponds to a way of being true, otherwise any possible non-empty extension of ' $x$ is a zombie' corresponds to a way of being false. In the following, I will use the general term witness to denote indistinctly ways of being true or ways of being false of a certain sentence. Alternatively, I may use the expression verifier (falsifier) to denote the witness associated with a way of being true (false).

Yablo's proposal may be summed up by means of the following criterion:

\section{Exact aboutness}

The sentence $\varphi$ is exactly about $m$ iff

- Worlds agreeing on the $m$-conditions also agree on the truth-value of $\varphi$, or, in other words, the subject matter $m$ subpartitions the $\varphi$-partitioning. (Truth-value supervenience)

- There is at least one $m$-cell that is a proper subset of an $\varphi$-cell (Non-minimality)

- There is at least one $m$-cell that is not a singleton (Non-maximality)

- Each $m$-condition corresponds to a way of being true (false) of $\varphi$ (where each way of being true corresponds to the list of verifiers (falsifiers) of $\varphi$ in some possible worlds)

In the next section all these notions will be used to define the carving of a content and the recarving operation. 


\section{CARVING UP A CONTENT}

Frege's account of the notion of content (Begriffinhalt) is not as clear as his account of other semantic notions, e.g. the germane notion of sense. This fact is easily explained if we take into account that he did not develop his mature theories on the ground of the notion of content; from 1884 on, this notion was progressively abandoned. Frege himself seems to suggest that the notion was somehow confusedly presented:

When I wrote my Foundations of Arithmetic, I had not yet made the distinction between sense (Sinn) and reference (Bedeutung); and so, under the expression 'the content of a possible judgement', I was combining what I now designate by the distinctive words 'thought' (Gedanke) and 'truth-value' (Wahrheitswert). (Frege et al., 1951)

The combination of notions mentioned in the passage has lead several scholars to suppose that the notion of content was something in between sense and reference 5 ; in particular, Hale (1997) proposes that the notion of content should be identified with what he calls weak sense, roughly speaking, weak sense is defined as some strong notion of truth-conditions which should stand for the state of affairs that a certain sentence describes. Though introducing an interesting perspective on the matter, this is not the position I take in this paper: I propose that two sentences are identical in content iff they bave the same truth-value in all possible worlds. If the proposition expressed by a sentence is taken to be the set of possible worlds in which the sentence is true, a consequence of my proposal is the identification of contents with propositions.

5 (Hale, 1997), (Dummett, 1991)

Manuscrito-Rev. Int. Fil. Campinas, v. 42, n. 1, pp. 45-90, Jan-Mar. 2019. 
The view that any two logically equivalent sentences have the same content seems at odd with some aspects, consequences, and interpretations of Frege's view:

1. Suppose that all necessary truths have the same content; yet if all theorems of arithmetic share the same content with all theorems of logic, how truths arithmetic may be informative without presenting any addition of content to truths of logic?

2. There seems to be a certain relation of relevance between the two sides of an abstraction principle; however, there are many cases of logically equivalent sentences that do not present any relevance at all. Thus identity of content should be a relation stronger than logical equivalence?

The first line of thought is based on the assumption that informativeness is characterized in terms of a difference in content, in particular, in terms of a certain surplus of content. I don't have an argument to reject this view; my only remark is that informativeness is an epistemic notion while content is not. Thus informativeness may be characterized in terms of the way a certain content is accessed and not in terms of content itself: for instance, a certain logical equivalence $\alpha \equiv \beta$ may be informative in virtue of the fact that the same content is presented in different ways and not in virtue of a difference in content between $\alpha$ and $\beta$.

The second point is raising a more fundamental objection to the identification of the content of a sentence

${ }^{6}$ A similar remark is made by M. Kremer in his essay induded in (Ricketts and Potter, 2010), p.220-293.

${ }^{7}$ I attribute this position to Hale (1997).

Manuscrito-Rev. Int. Fil. Campinas, v. 42, n. 1, pp. 45-90, Jan-Mar. 2019. 
with the proposition it expresses. My reply is that as far as the main purpose of the analysis is not merely exegetic, the difficulty is mainly terminological. Once the interpretation of Frege's notion of content is not a priority, what matters is the characterization of the relation of relevance holding between the two sides of an abstraction principle; according to my proposal, this is a relation that holds between some special carvings of a certain content: call this family of carvings related carvings; according to what may be called the fine grained content approach, the relation of relevance holds between two sentences in virtue of the fact that they are carvings of the same content. Suppose now that there is a valid argument to the effect that Frege's notion of content should not be identified with that of proposition: once the relation of relevance has been satisfactorily characterized, one may decide to simply call 'proposition' what I call 'content' and to say that two sentences are identical in content whenever I say that they are related carvings ${ }^{8}$. On the other hand, the identification of content with proposition allows me to discuss the issue within a better understood theoretical setting; this may result in an interpretation of the notion of carving and recarving easily understandable also outside of the context of Frege's project.

I will now introduce the needed definitions to formulate my proposal. Let $\mathbf{W}$ be the class of all possible worlds; I will assume that $\mathbf{W}$ is associated with a certain interpretation of a certain language. I define the bilateral proposition expressed by a sentence $\varphi$ - denoted by $\quad|\varphi|$ - in the following way:

$$
|\varphi|=\left\langle|\varphi|^{T},|\varphi|^{F}\right\rangle
$$

8 When the operation of recarving will be defined, instead of using the expression 'related carvings' I will say that two sentences are one the recarving of the other. 
where $|\varphi|^{T}\left(|\varphi|^{F}\right)$ stands for the set of possible worlds in which $\varphi$ is true (false). I take the content of $\varphi$ to be the bilateral proposition expressed by $\varphi$. Thus, the content of a sentence is something very close to its truth-value, or, in different words, is a minimal notion of truth-conditions of a sentence, i.e. what is commonly known as the intension of the sentence 9 . In a preliminary way, I may say that the content of a sentence $\varphi$ conveys no more information than the truth-table of $\varphi$.

Even if the content of a sentence has been characterized in purely extensional terms, there are different ways of understanding the given definition. For instance, for the purpose of the following exposition, the content of a sentence $S$ may be conceived as the characteristic function associated with $S$, i.e. the function that returns the value 'True' when applied to a world according to which $S$ is true, the value 'False' otherwise. When the content is thus understood, I will also speak of the content as a condition on possible worlds, where the word 'condition' has to be intended in its extensional sense, i.e. as a characteristic function. Given a sentence $S$, the condition associated with the content of $S$ may be determined by the expression 'the world $w$ is such that the sentence $S$ is true'.

Albeit the given definition of content may result very simple, it becomes problematic when the task of defining the carvings of a content is considered. Indeed, there is no immediate sense we may give to the expression 'carving up an ordered pair of sets of possible worlds in different ways'. Even if we assume that to carve up a set is to divide it into proper subsets, sets give us no hint in how they should be

9 Hale (1997) also identifies content and truth-conditions, yet he seems to have in mind a more refined notion of truth-conditions; for this reason I use the adjective 'minimal' to characterize the notion of truth-table-like truth-conditions. 
divided. More specifically, we may allow decompositions of each set $A, B$ composing the content $\langle A, B\rangle$ into proper subsets as carvings; however there are three main difficulties related to this approach: 1) Given a content $\langle A$ , $B>$ it must be clarified what is the condition that determines a certain decomposition; 2) it is not clear what such decomposition should mean 3 ) it is even less clear what a decomposition of sets into proper subsets has to do with the description of Frege's procedure. My claim is that these difficulties may be overcome by applying LewisYablo theory of subject matter to the notion of content carving as preliminary understood.

It has been said that a certain content may be associated with a certain condition on possible worlds. Consider now the sentence $\varphi$ being Madison Ave is parallel to the 5th Avenue' and let $c$ be its content; for each possible world $w$, $c$ tells us whether $w$ makes $\varphi$ true or not; consider now the way $c$ seems to be carved up by $\varphi$ : for each possible world $w$, it is said whether two constituents of $w$, i.e. Madison Ave and the 5th Avenue, are brought together in such a way that $\varphi$ is true or not. This simple consideration shows that a carving of a content may be seen as the conversion of a condition on worlds into a new condition applied to worldconstituents (or, to be general, to some specific aspects of a world). In other words, while the content $c$ tells us whether a world $w$ meets a certain requirement $R$ or not, a carving of $c$ tells us how a world $w$ meets $\mathrm{R}$.

According to the given definition, the content of a sentence $\varphi$ distinguishes between worlds that make $\varphi$ true and worlds that make $\varphi$ false. The way $\varphi$ carves up this content traces a finer distinction: among worlds that makes $\varphi$ true (false) it distinguishes worlds that make $\varphi$ true (false) in different ways. In other words, a content partitions the logical space into the region of truth and that of falsity, while a carving of a content partitions the logical space into ways of being true and ways of being false. This takes us to 
the notion of exact subject matter: a sentence $\varphi$ carves up its content $c$ according to what $\varphi$ is exactly about. More precisely, $\varphi$ partitions the region of truth (falsity) of $c$ into sub-cells univocally associated with $\varphi$ 's ways of being true (false).

I have previously mentioned that to carve up the content of a sentence $S$ is to convert the condition on possible worlds determined by $S$ into a condition on worldconstituents, where the word 'condition' in the first occurrence has to be intended in an extensional sense, i.e as the characteristic function of the sentence (or, in Carnap's terminology, intension). How the word 'condition' should be understood when applied to world constituents? We may define also this new condition as an extensional truth function. Let $S$ be a sentence and $m$ the exact subject matter of $S$. As Yablo himself suggests, we may conceive the subject matter $m$ as a function that given a possible world $w$ returns the witness of $S$ in $w$. For instance, given that the sentence 'Socrates is wise' is exactly about Socrates, the function $m$ associates to every possible world $w$ the extension of the proper name 'Socrates' in the world $w$. Hence we may define the condition introduced in the informal definition of content carving as a function - say it $F_{S}$ - defined over all witnesses and having the set of truth-values as codomain. More precisely, given a witness $A, F_{S}(A)$ is the value 'True' if all worlds agreeing that the value of $m$ is $A$ makes the sentence $S$ true, otherwise $F_{S}(A)$ is the value 'False'.

According to the previous remarks, we may consider a carving of a certain content $c$ of a sentence $\varphi$ as the specification of the exact subject matter of $\varphi$, or, as a subpartitioning of $c$ according to the ways of being true and the ways of being false of the sentence $\varphi$. This definition may be easily formalized:

\section{Content Carving (CC)}


Let $\equiv_{\varphi}^{T}\left(\equiv_{\varphi}\right)$ be the relation that holds between two possible worlds whenever $\varphi$ is true (false) in the same way in them; let $\chi(\varphi)$ denote 'the way $\varphi$ carves up its content'; according to the given considerations:

$$
\chi(\varphi)=\left\langle|\varphi|^{T} / \equiv_{\varphi}^{T},|\varphi| F / \equiv_{\varphi}^{F}\right\rangle
$$

where $|\varphi|^{T /} \equiv_{\varphi}^{T}$ stands for the quotient set of $|\varphi|^{T}$ with respect to the equivalence relation $\equiv_{\varphi}^{T}$.

Given that all logically equivalent sentences have the same content, yet they may have different exact subject matters, there may be different carvings of the same content. One may ask if there is a purely extensional criterion that given the content $c$ of a certain sentence, allows us to determine all possible carvings of $c$, we may attempt the following definition:

\section{Criterion for content carving (CCC)}

Let $\langle A, B\rangle$ be a content; the pair $\langle K, H\rangle$ is a carving of $\langle A, B\rangle$, in symbols:

$$
\Gamma(\langle A, B\rangle,\langle K, H\rangle)
$$

iff the following conditions obtain:
(i) $\cup K=A \quad \& \quad \cup H=B$
(ii) $\forall x \in K(x \subset A \& x \neq \emptyset)$
(iii) $\forall x \in H(x \subset B \& x \neq \emptyset)$
(iv) $\exists x \in(K \cup H) \exists u, v \in x(u \neq v)$
(v) $\forall u, v \in(K \cup H)(u \neq v \Rightarrow u \cap v=\emptyset)$

Conditions (i)-(v) ensure that the set $K(H)$ is a decomposition of $A(B)$ into cells such that all $K$-cells $(H$ cells) are non-empty proper pairwise disjointed subsets of $A(B)$ and at least one element of $K$ or $H$ is not a 
singleton. According to the notions introduced in the previous section, conditions (i)-(v) ensure that the partitioning represented by $\langle K, H\rangle$ is associated with a genuine subject matter of the bilateral proposition $\backslash A, B$ ).

To see (CCC) as defining a carving is to assume that every genuine subject matter of a certain bilateral proposition is the exact subject matter of some sentence. This seems to be too strong: it implies that every combinatorially possible partitioning of a content satisfying conditions (i)-(v) defines a class of ways of being true (false), i.e. defines a class of possible verifiers (falsifiers). Under the assumption that a verifier $v$ is an entity which exists in all worlds in which the sentence that it verifies is true in the way associated with $v$, (CCC) implies a form of ontological inflationism. For we may introduce new entities with extreme liberty: it suffices only to partition a certain content in the suitable way defined by (i)-(v).

This consequence is highly undesirable; for now I say that not all combinatorially possible "re-partitionings" that satisfy condition (i)-(v) are to be considered as carvings of the given content. In section 5 I will attempt to characterize this restriction.

As previously suggested, from definition (2) it follows that two sentences are different ways of carving up the same content iff they are logically equivalent and they have different exact subject matters. Take the case of lines and directions: the sentence 'In the city $\mathrm{X}$, the street of the bakery is parallel to the street of the library ' 10 is exactly about the street of the bakery and the street of the library in the city $\mathrm{X}^{11}$, whereas the sentence 'In the city $\mathrm{X}$, the

10 Assume that in the city $\mathrm{X}$ there is only one bakery and only one library.

11 Firstly, note that worlds agreeing on which streets the street of the bakery and the street of the library are, must agree on whether

Manuscrito-Rev. Int. Fil. Campinas, v. 42, n. 1, pp. 45-90, Jan-Mar. 2019. 
direction of the street of the bakery is identical to the direction of the street of the library' is about different things, i.e. directions (supposing for simplicity that the existence of directions is uncontroversial). To see the difference, note that two possible worlds may agree on the common direction of the street of the bakery and the street of the library (for instance they may agree that the two streets form a certain angle $\theta$ with the north-south axis) while disagreeing in which street the bakery or the library is (the two streets may be different from a world to another while maintaining the same direction). Moreover, it is impossible that there are two worlds assigning the same direction to the streets and different truth-value to the sentence asserting the identity of these directions, thus directions are a genuine subject matter of the considered sentence. Moreover, it seems to be absurd to allow the sentence to be true (false) in the same way when the directions of the considered streets has changed from one world to another; thus the sentence is exactly about the direction of the street of the bakery and the direction of the street of the library.

I hope to have successfully showed how to overcome two of the three difficulties related to the notion of carving when applied to a set theoretic definition of content. The first difficulty was related to the determination of the conditions that decompose a set into proper subsets, the second to the requirement of a meaningful interpretation of

they are parallel or not. Moreover, recall Yablo's remark on the sentence 'The U.S. president in 2001 is a senator's son': a change in the entities with which a certain subject matter is concerned must imply a change in the way the sentence is true. Thus, a transworld change in the streets for which 'the street of the bakery' and 'the street of the library' stand that maintains them parallel, is a change of the way the sentence asserting their parallelism is true.

Manuscrito-Rev. Int. Fil. Campinas, v. 42, n. 1, pp. 45-90, Jan-Mar. 2019. 
such a decomposition. According to my proposal, given a sentence $\varphi$, the true (false) region of the logical space is partitioned according to $\varphi$ 's ways of being true (false); thus ways of being true (false) represent the condition for decomposing the true (false) region into proper subsets. It has been shown that this decomposition is associated with a philosophically significant notion, i.e. the notion of exact subject matter. There is still a third difficulty, i.e. the relation between the proposed decomposition and Frege's procedure; it will be solved in the next section, when the operation of content recarving will be defined.

Given the identification of the content of a sentence with its minimal truth- conditions, an interesting question would be whether the carving of a certain content may be identified with a certain notion of refined truth-conditions. The answer is positive: it suffices to see that the operation of carving up a content consists in the transformation of a condition on worlds into a new condition on the specific aspect of worlds which is determinant for the truth-value. Thus by carving up the content of a sentence $\varphi$ we pass from a "brute expression of the truth-conditions" to a more refined version, in which we specify some constituents/aspects of a possible world that brought together in a suitable way make $\varphi$ true.

One may object that my proposal on content carving does not take into account another aspect of the preliminary understanding of the notion: the logical form. Indeed, one may understand the fact that two sentences are different ways of carving up the same content as the fact that they are logically equivalent sentences having different logical forms. An immediate reply to the objection would be that the language in which the sentences are expressed must be regimented in such a way that a difference in subject matter is a necessary and sufficient condition for a difference in logical form. Yet this is impossible; consider the sentence $\varphi$ and the sentence $\varphi \wedge(\varphi \vee \neg \varphi)$ and assume that $\varphi$ is exactly 
about $m$ : both $\varphi$ and $\varphi \wedge(\varphi \vee \neg \varphi)$ are exactly about $m$ while having different logical forms. Thus to assume that a logical form picks out a unique carving of a certain content is in contrast with the definitions given in this section.

I don't have a definitive argument to the effect that a syntactic criterion for carving distinction in terms of logical form is less satisfactory than a semantic one in terms of subject matter. To my mind, when the notion of content carving is concerned, the notion of logical form is interesting insofar as it provides information about the way truth-conditions are satisfied. On this aspect, the sentence $\varphi$ $\wedge(\varphi \vee \neg \varphi)$ does nothing more than $\varphi$, for the conjunct $\varphi$ $\vee \neg \varphi$ does not even contribute to the determination of the truth-value of $\varphi$.

In conclusion, I remark that given a certain carving, i.e. given a certain refined expression of the truth-conditions of a sentence, there are logical forms that naturally correspond to this expression (as in the example of parallelism and identity of directions) and logical forms that expresses the same refined truth-conditions in a very roundabout way (as in the example of the previous paragraph). According to my proposal, the latter cases collapse into the former ones, for less natural cases of logical form fail to specify a different exact subject matter ${ }^{12}$. As a consequence, my notion of content carving is insensitive to artificial syntactic constructions that are not determinant for the assignment of truth-value. In this sense, my account is less dependent on language than the view according to which each logical form is univocally associated with a carving.

12 A different and equally interesting approach to the matter is Yablo distinction between invited and uninvited recarvings, (Yablo, 2008). 


\section{CONTENT RECARVING}

In this section the part of Frege's procedure labeled 'content recarving' will be analyzed and defined. We will start by highlighting some similarities between the operation of carving up a content and that of recarving a content already carved up in a certain way.

Consider Frege's example: it has been said that the sentence ' $a \|$ l| $b$ ' carves up its content according to what it is exactly about, i.e. lines $a$ and $b$; more specifically, the carving associated with ' $a \| \quad b$ ' is the transformation of a condition on worlds into a new condition - determined by the relation of parallelism - on relevant constituents of worlds, i.e. the lines for which the expressions ' $a$ ' and ' $b$ ' respectively stand. In this way, we refine the given truth-conditions by focusing on a specific aspect of a world and not on a world in its entirety. We may conceive content recarving as a similar operation: we transform a condition on some constituents of a world into a new condition on some more relevant aspect of these constituents. For instance, we transform the condition of parallelism between lines into a new condition, i.e. identity, applied to an aspect of lines, i.e. directions, that is the most relevant when parallelism is concerned. These considerations may be taken to go toward the fundamental idea of my proposal: the operation of content recarving is an operation of restriction of a certain subject matter.

The similarity between carving and recarving may illuminate further aspects of Frege's procedure: the restriction of our attention on a relevant aspect of lines may be seen as the result of applying the specific part of the content of the relation of parallelism to its relata; the new conditions obtained over directions may be seen as the result of removing the specific part of the content of the original condition. These two aspects are in some sense complementary: when we restrict our attention on a specific 
aspect of lines we automatically make the condition applied to lines more general, i.e. we transform it into an identity. This fact may be spelled out as follows: an equivalence relation $R$ may be seen as a partial identity: in fact, every equivalence relation defines a class of invariant properties, i.e. all properties that two object that are $R$-related have in common. As a consequence, two objects are R-related iff they have all the $R$-invariant properties in common. This may be seen as a restricted version of Leibniz's law: whenever two objects shares all properties that are in a certain class $\Delta$, they are partial identical (or identical with respect to $\Delta$ ). According to this reading of an equivalence relation, what is specific of the content of $R$ is the class of invariant properties it defines; and all invariant properties characterize the aspect of the relata that is relevant to the equivalence relation. Thus to remove what is specific in the content $R$ is to convert a partial identity into a total identity, and to apply what is specific of $\mathrm{R}$ to $a$ and $b$ is to pick out the aspect of $a$ and $b$ associated their R-invariant properties. In the case of lines, we may say that the concept of direction is determined by the class of all orientational properties defined by the relation of parallelism; in the case of concepts, the concept of cardinal number is determined by the class of quantitative properties associated with the relation of equinumerousity.

One may object that the previous explanation does not help to understand the operation of content recarving insofar as it lacks generality, for it is relevant just to the case of abstraction principles. Though the objection is correct, it is possible to extrapolate a pattern of explanation that apply to all cases. To this end, consider the following example of content recarving:

let $\varphi$ be the sentence 'The U.S. president in 2017 is blond (blonde)'; it is exactly about the U.S. president in 2017. We may recarve this content by restricting the subject matter the U.S. president in 2017 and obtain the hair color of the U.S. 
president in 2017 ; if we do this, we obtain the sentence $\psi$ : 'The hair color of the U.S. president in 2017 is in the rang of yellow'13. It is easy to see that the new sentence is exactly about the hair color of the U.S. president; it can also be shown that the hair color of the U.S. president in 2017 is a subject matter strictly included in the U.S. president in 2017, for all worlds wholly agreeing on who's the U.S. president in 2017 must agree on his/her hair color, yet not all worlds agreeing on the hair color of the U.S. president in 2017 also agree on who's the president. As a result of such a subject matter restriction, the condition ' $x$ is blond (blonde)' has now been generalized so that the condition ' $x$ is in the range of yellow' obtains; to be blond (blonde) is a property that concerns the color of a specific part of a person, while to be in the range of yellow applies to all object having a defined color.

There is a general way to present this fact. Given a sentence $\varphi$, we may always distinguish between the subject matter of $\varphi$ and what $\varphi$ says about its subject matter. Let $\psi$ be a sentence that recarves the content of $\varphi$ by restricting the subject matter $m$ of $\varphi$ in such a way that a new smaller subject matter $n$ obtains. Consider now an $n$-cell $A$ associated with a certain witness $a$ of $\psi$. Given that $n$ is strictly included in $m$, there is always an $n$-cell that may be seen as obtained by merging some (at least two) $m$-cells. Suppose that the $n$-cell $A$ is the result of merging the $m$ cells $B_{1}, B_{2}$, and $B_{3}$ to which the witnesses of $\varphi b_{1}, b_{2}$, and $b_{3}$ are associated. The fact that what $\psi$ says about $n$ is more general than what $\varphi$ says about $m$ may be explained thus: we may describe a certain circumstance in which $\psi$ is true by saying that $a$ is compatible with what $\psi$ says about $n$; this very circumstance corresponds to three different circumstances in which $\varphi$ is true, viz. the circumstances in

13 Assuming that it is a necessary truth that whenever someone is blonde her hair color is in the range of yellow.

Manuscrito-Rev. Int. Fil. Campinas, v. 42, n. 1, pp. 45-90, Jan-Mar. 2019. 
which either $b_{1}$ or $b_{2}$ or $b_{3}$ is compatible with what $\varphi$ says about $m$. Therefore what $\psi$ says about $n$ is more general because with a single condition of compatibility it captures a multiplicity of circumstances in which what $\varphi$ says about $m$ is the case. Or, in different terms, for an object $a$ to be compatible with what $\psi$ says about $n$ less is required from a possible world than what is required for $b_{1}\left(b_{2}\right.$ or $\left.b_{3}\right)$ to be compatible with what $\varphi$ says about $m$.

Thus content recarving may be conceived as a content preserving operation that by making more specific one aspect of the content (i.e. the subject matter of the given sentence) has to make more general the other (i.e. what the given sentence says about its subject matter). These two aspects of the same operation may be associated with the two steps of Frege's procedure: the determination of a more specific subject matter may be associated with the division of the specific content of the relation of parallelism between the two lines; the operation of removing the specific content of the relation of parallelism may be associated with the generalization of the condition applied to the original subject matter.

Having clarified the general idea of content recarving, it is possible to formulate a rigorous definition:

\section{Content recarving (CR)}

Let $\varphi$ and $\psi$ be sentences; let $\chi(\varphi)$ and $\chi(\psi)$ be the respective carvings; we say that $\chi(\psi)$ is obtained by recarving $\chi(\varphi)$, in symbols

$$
\Theta(\chi(\psi), \chi(\varphi))
$$

iff

(i) $|\varphi|=|\psi|$

Manuscrito - Rev. Int. Fil. Campinas, v. 42, n. 1, pp. 45-90, Jan-Mar. 2019. 
(ii) $\forall x \in\left(|\varphi|^{\mathrm{T}} / \equiv^{\mathrm{T}} \cup|\varphi| \mathrm{F} / \equiv_{\varphi} \mathrm{F}_{\varphi}\right) \forall y \in\left(|\psi| \mathrm{T} / \equiv^{\mathrm{T}} \mathrm{T}\right.$ $\left.|\psi| \mathrm{F} / \equiv \mathrm{F}_{\varphi}\right),(x \subseteq y \vee x \cap y=\emptyset)$

(iii) $\exists x \in\left(|\varphi|^{\mathrm{T}} / \equiv^{\mathrm{T}} \cup|\varphi| \mathrm{F} / \equiv_{\varphi} \mathrm{F}_{\varphi} \exists y \in\left(|\psi| \mathrm{T} / \equiv \mathrm{T}_{\varphi} \cup\right.\right.$ $\left.|\psi| \mathrm{F} / \equiv \mathrm{F}_{\varphi}\right),(x \subset y)$

Some remarks on the given definition. Firstly, the formula $\Theta(\xi, \zeta)$ has the metavariables $\xi$ and $\zeta$ ranging over carvings, yet it may also be read as a relation between sentences, by defining

$$
\Theta^{*}(\psi, \varphi) \Leftrightarrow \Theta(\chi(\psi), \chi(\varphi))
$$

for this reason we may simply say that $\psi$ is a recarving of $\varphi$ whenever the way $\psi$ carves up its content is obtained by recarving the way $\varphi$ carves up its content. The definition (CR) says that $\psi$ is a recarving of $\varphi$ whenever $\varphi$ and $\psi$ are logically equivalent and the exact subject matter of $\psi$ is strictly included in the exact subject matter of $\varphi$. By simple set theoretic considerations it is possible to conclude that $\Theta$ is a partial strict order, i.e. it is an anti-reflexive, antisymmetric, and transitive relation. It is interesting to see that $\Theta$ is not connected, i.e. there are carvings of the same content such that none is the recarving of the other. This is explained by the fact that there may be logically equivalent sentences whose subject matters are not in a relation of inclusion, for instance in the case that the intersection between a cell of one subject matter is non-empty and different from a cell of the other subject matter. This result is in line with one aspect of our preliminary understanding of the notion of content recarving: for $\psi$ to be a recarving of $\varphi$, a certain relation of relevance between $\varphi$ and $\psi$ is expected: my proposal rigorously defines such a relation of relevance as strict inclusion of subject matter. 
From a set theoretic point of view, (CR) provides an interesting characterization of the relation of recarving conceived as an operation. Let $m$ be the exact subject matter of a certain sentence, and $n$ the subject matter resulting from the recarving. Clearly, $n$ is strictly included in $m$; by definition of subject matter inclusion, every $m$-cell is a subset of every $n$-cell with at least one $m$-cell being proper subset of one $n$-cell. As a consequence, the restriction of subject matter associated with content recarving may be seen as performed by merging some $m$-cells to obtain the $n$ cells.

Content recarving has been defined as a relation between carved contents; the previous remark suggests that it may also be conceived as an operation performed on a carving, provided that the new restricted subject matter is given in advance. Let $\varphi$ be a sentence and $m$ a subject matter; denote by $[\varphi]$ the subject matter of $\varphi$; the operation of recarving according to the subject matter $m$, in symbols $\Theta_{m}$ is defined as follows:

$$
\Theta_{m}(\chi(\varphi))=\langle K, H\rangle
$$

iff

(i) $m<[\varphi]$

(ii) $\Gamma(|\varphi|,\langle K, H\rangle)$

(iii) $K=|\varphi|^{\mathrm{T}} / \bar{\Xi}_{m} \quad \& \quad H=|\varphi| \mathrm{F} / \equiv_{m}$

where the expression ' $m<[\varphi]$ ' stands for 'the subject matter $m$ is strictly included in the subject matter of $\varphi$ ', the relation $\Gamma$ is the one defined in (CCC), and ' $\equiv_{m}$ ' stands for the equivalence relation associated with the subject matter $m$ (i.e. the relation that holds between two worlds whenever they are indistinguishable with respect to $m$ ).

Given a subject matter $\mathrm{m}$, the result of the operation $\Theta_{m}$ may not be the carving of a defined 
sentence but just a hypothetical carving. This because nothing ensures that the cells in $K(H)$ correspond to ways of being true (false) of a certain sentence; in other words, we have no guarantee that what is indistinguishable between worlds belonging to the same cell in $K(H)$ is an existing witness of some sentence $\psi$. This leads to fundamental semantic and metaphysical issues related to the notion of content recarving: the discussion on these aspects of the notion will be the focus of the next section.

\section{RECARVING AT THE JOINTS}

The account of the operation of content recarving given so far left unanswered some fundamental questions on the matter: when the result of a recarving obtained by a mere merging of partitions effectively corresponds to a new subject matter of a new sentence? What is the ontological import of such an operation?

These questions point at the problem of distinguishing between merely hypothetical carvings, i.e. carvings obtained by a mere set theoretic procedure, and effective carvings, i.e. carvings that besides being the result of a certain merging also present some kind of connection with the metaphysical structure of reality. To trace this distinction two problems must be solved: 1) the problem of the determination of the new restricted subject matter associated with a recarving operation; 2) the problem of establishing the ontological import of the new ways of being true (false) of the new sentence exactly about the restricted subject matter. For instance, in the case of Frege's example, the solution of the first problem would be the answer to the question "why directions?" and the solution of the second problem would be the answer to the question "Given that we may restrict the subject matter 
line $a$, line $b$ to direction of $a$, direction of $b$, on what grounds we may say that directions exist? ". In the following part of this section I will propose a sketch of a solution to the first problem (determination of the new subject matter); regarding the second issue, I will limit myself to specify in which terms the problem may be formulated without disputing the details.

Given a sentence $\varphi, \varphi$ carves up its content according to its exact subject matter; for instance, the subject matter U.S. president in 2017 divides the logical space into cells each of which is associated with a certain individual. There is a huge variety of possible ways of merging these cells in order to obtain a coarser partitioning of the logical space; how ever, many of them are merely arbitrary insofar as they are just the result of set theoretic operations. If we want a guiding criterion that tells us which cell should be merged with which, then we need a certain pattern of resemblances between the ways the sentence whose content is to be recarved is true (false); and it is reasonable to assume that such a pattern of resemblances is given by a battery of necessary truths related to the subject matter at issue. For instance, given the subject matter U.S. president in 2017, consider the cell associated with the actual Donald Trump (i.e. the set of worlds in which the actual Donald Trump is the U.S. president in 2017) and that associated with the actual Hilary Clinton. If we want to merge the Trump-cell with the Clinton-cell, we should do this on the ground of a certain resemblance between Clinton and Trump that instantiates a certain general pattern of resemblances between U.S. presidents in 2017; given that the required relation of resemblance has to do with entities of different possible worlds, it depends on a certain class of necessary truths about

U.S. presidents in 2017 (for instance the set of truths analytic of the concept of U.S. president or of the concept of person). However, not any sort of resemblance may serve 
our purpose: the specific resemblance between Clinton and Trump must be relevant to what the original sentence (i.e. the sentence to be recarved) says about the subject matter U.S. president in 2017 ; for example, if the sentence is 'The U.S. president in 2017 is blond/blonde', then the Clintoncell and the Trump-cell may be merged iff there is a relation between Clinton and Trump that instantiates a pattern of resemblances with respect to blondness.

The goal of determining the new subject matter resulting from the recarving may be achieved in two steps: firstly, a criterion for saying when a certain partitioning of the logical space corresponds to an effective subject matter, i.e. a partitioning of the logical space such that all worlds belonging to the same cell must have something in common, in the sense of being indistinguishable under a certain respect; secondly, once the aforementioned pattern of resemblances has been defined, it must be shown that the new subject matter determined by the pattern of resemblances is a subject matter satisfying the criterion of effectiveness of the previous point. As it turns out, both the criterion of effectiveness and the definition of the pattern of resemblances are based on the same notion: the relation between a determinable property and its determinate properties.

The relation determinable-determinates may be briefly described as a particular kind of specification relation: 'red' (i.e. the determinate) is a specifier of 'colored' (i.e. the determinable), 'having $2 \mathrm{~kg}$ of mass' is a specifier of 'having mass', 'having a length of two meters' is a specifier of 'having a length' etc. Intuitively, each cell in which a subject matter divides the logical space seems to be a specifier of the same sort: consider the subject matter 'The US president in 2017'; it partitions the set of worlds according to which there is a US president in 2017. Thus we may see this set as the extension of the determinable property ' $w$ is a world according to which there is a US president in 2017'. 
Correspondingly, each cell is the extension of a certain determinate: for instance, the cell including all worlds according to which the US president in 2017 is the actual Donald Trump may be seen as the extension of the property ' $w$ is a world according to which the actual Donald Trump is the US president in 2017'.

The fundamental idea is to take the relation determinate-determinable as a characteristic feature of effective subject matters, i.e. of subject matters that are not just the result of a mere set theoretic operation of partitioning. Yet to do this, we need a criterion for saying when a class of properties may be considered the determinates of a certain determinable.

According to the relevant literature, the fundamental features that characterize the relation determinatedeterminables are the following:

i. (Exclusiveness) If $Q$ and $M$ are determinates of $P$, then no object having $P$ may have both $Q$ and $M$ ((Johnson, 2014), (Prior, 1949))

ii. (Non-conjunctive) If $Q$ is a determinate of $P$, then $Q$ is a non-conjunctive specifier of $P$ and it is logically related to any non-conjunctive specifier of $P$ , where two properties are logically related whenever either one entails the other or one entails the negation of the other (Körner and Searle, 1959)

iii. (Modal constraint) If $Q$ is a determinate of $P$, then necessarily to have $Q$ entails to have $P$ and possibly am object may have $P$ without having $Q$. (Yablo, 1992)

iv. (Levels) There possibly are many levels of specification of $P$, each level corresponding to a different class of determinates of $P$; every determinate of $P$ of a certain level is a determinable with respect to a certain class of determinates of $P$ of the next level.((Johnson, 2014), (Prior, 1949)) 
To understand how these four conditions apply, consider the most common example of the relation of determination, i.e. the one holding between a particular color property and the property of being colored. (Exclusiveness) is satisfied, for no object may have two different colors at the same time. To understand (Non-conjunctive) we need to spell out the notion of 'non-conjunctive specifier'; for instance, the property of being human is a specifier of the property of being animal, for not all animals are humans. However, human is a conjunctive specifier, for there is a property not necessarily co-extensional with human, i.e. rational, such that the conjunction of this property with the property of being an animal is co-extensional with the property of being a human. It is easy to see that ' $x$ is red' cannot be defined as the conjunction of ' $x$ is colored' and some other property not co-extensional with ' $x$ is red'. In other words, the relation between a determinable and a correspondent determinate does not reduce to the relation between genus and specie. Moreover, given any other non-conjunctive specifier of ' $x$ is colored', for instance ' $x$ is yellow', ' $x$ is red' implies that ' $x$ is not yellow'. (Modal constraint) is easy to understand: necessarily, if something is red, then it must be colored, yet something might be colored without being red. (Levels) is one of the most interesting feature: ' $x$ is red' is a determinate of ' $x$ is colored' while it is a determinable with respect to the determinate ' $x$ is scarlet'.

What the relation of determination has to do with the notion of subject matter? I will not propose a general argument to the effect that the existence of a relation of determination between properties of possible worlds is a necessary and sufficient condition for the effectiveness of a subject matter; nonetheless, I will try to convince the reader that this is the case by referring to some examples.

Consider the subject matter 'The US president in 2017'. It is an effective subject matter, for worlds agreeing on the 
US president in 2017 do have something in common: they are partially indistinguishable, i.e. indistinguishable with respect to the US president in 2017. We will show that for every $X$ such that $X$ is a possible US president in 2017 , the cell associated with $X$ is a determinate of the determinable property ' $w$ is a world according to which there is a US president in 2017'. Firstly, note that no world may admit both the actual Donald Trump and the actual Hilary Clinton as US presidents, which proves that (Exclusiveness) holds ${ }^{14}$.

Regarding (Non-conjunctive), it is hard to imagine how the property ' $w$ is a world according to which the actual Donald Trump is the US president in 2017' may be defined by putting the property ' $w$ is a world according to which there is a US president in 2017' in conjunction with another property not co-extensional with the former. Indeed, it seems that such property may be defined by the conjunction of all properties of the sort ' $w$ is a world according to which the US president in 2017 is $F$ ' with $F$ intrinsic property of the actual Donald Trump; yet this conjunctive property is co-extensional to ' $w$ is a world according to which the actual Donald Trump is the US president in 2017'. (Modal constraint) is straightforward: necessarily, if $w$ is such that the actual Donald Trump is the US president in 2017, then $w$ must be a world according to which there is a US president in 2017; moreover, from the

14 One may disagree by supposing that there is a possible world in which the United States in 2017 have adopted a sort of duumvirate, thus admitting two presidents. Yablo himself ((Yablo, 2014), p.36) recognizes the possibility of subject matters that are not defined by an equivalence relation due to the lack of transitivity, as in this case. For sake of simplicity, I will not consider this sort of subject matters in the present discussion; it is as if the operation of recarving may be applied only to subject matter that are in good order, i.e. that partitions the logical space into disjointed cells. 
fact that $w$ is a world according to which there is a US president in 2017, it does not always follow that, according to $w$, the US president in 2017 is the actual Donald Trump. (Levels) also is satisfied, and it is the most interesting feature for - as we will see - is what makes the recarving operation possible. Let $m$ be an effective subject matter and $n$ a subject matter strictly included in $m$; it is easy to show that each $m$-cell is a determinable having as determinates all $n$ cell that it strictly contains. Let $m$ be the subject matter 'The hair of the US president in 2017' and $n$ the subject matter 'The US president in 2017'; by a reasoning similar to the one previously given, it is possible to show that the property ' $w$ is a world according to which the object $a$ is the hair of the US president in 2017' is a determinable having of the property ' $w$ is a world according to which the object $a$ is the hair of the actual Donald Trump'.

The relation determinate-determinable may be reasonably taken also as a sufficient condition. To see this point it suffices to note that the way a set of determinates partitions the domain under consideration defines a relation of partial indistinguishability with respect to the determinable ${ }^{15}$. Indeed, all red things are indistinguishable with respect to their color, as well as a red thing and a yellow thing are distinguishable - inter alia - under the same respect. As suggested by Lew is (1988), what I have called an effective subject matter has the characteristic feature of being defined by an equivalence relation that is a relation of partial indistinguishability under a fixed respect.

Therefore the following criterion may be reasonably assumed:

${ }^{15}$ A similar remark is made in (Armstrong, 1996), pp.51-55.

Manuscrito-Rev. Int. Fil. Campinas, v. 42, n. 1, pp. 45-90, Jan-Mar. 2019. 


\section{Criterion for effectiveness of a subject matter (CESM):}

Let $m$ be a subject matter and $\boldsymbol{W}$ be the set on which $m$ is defined. The subject matter $m$ is effective iff every $m$-cell is the extension of a determinate of a determinable having $\boldsymbol{W}$ as extension.

Note that the fact that a subject matter $m$ is effective does not imply that to each $m$-cell $A$ corresponds an object existing in all worlds belonging to $A$ that is a witness for every sentence exactly about $m$; it simply says that all worlds in $A$ are indistinguishable with respect to $m$, not that they must have a part in common.

The criterion (CESM) is used to determine when a certain partitioning of the logical space is an effective subject matter. Nonetheless, what we require to perform a recarving operation is something more: given a sentence and its exact subject matter, we want a procedure that determines a new effective subject matter strictly included in the initial one; or at least, we want a procedure that determines a certain range of effective smaller subject matters.

As previously mentioned, we may do this according to a certain pattern of resemblances between the witnesses of the given sentence, a pattern that must display a certain sort of relevance with what the sentence says about its exact subject matter. In the following we will see how to define this pattern.

Let $S$ be the sentence 'The US president in 2017 is blond' and let $m$ be its exact subject matter 'The US president in 2017'. As briefly mentioned in section 3, we may represent the intension $\mathrm{C}_{S}$ of $S$ as obtained by a functional composition between the subject matter function $m$ and the truth-function $F_{S}$ representing the condition in the way $S$ carves up its content, i.e 
for all $w \in \mathbf{W}, \mathrm{C}_{S}(w)=F_{S}(m(w))$

where $F_{S}$ may be seen as the function that, for every object $x, F_{S}(x)$ is the value 'True' iff $x$ is blond, and $m$ is the function that associates to every possible world $w$ the witness of $S$ in $w$.

Again, we may use the relation of determination between properties to define the pattern of resemblances and to grant that the new restricted subject matter is an effective one. Let $U_{S}$ be the set of all witnesses of $S$; under the assumption that there is a determinable property $P$ shared by all witnesses in $U_{s}$, we may say that two witnesses stand in the required resemblance relation iff they have the same determinate of $P$. Moreover, we must require that the resemblance relation is relevant to what $S$ says about $m$; thus we add the following condition: for every two witnesses of $S u$ and $v$, and for every determinate $X$ of $P$, if $u$ and $v$ both have $X$, then $F_{S}(u)=F_{S}(u)$, namely, if two witnesses stand in the relation of resemblance defined by the determination of $P$, then either both witness the truth of $S$ or both its falsity (either both are ways of being true of $S$, or both are ways of being false). With reference to the considered example, the determinable property may be ' $x$ is not bald' and each determinate may be ' $x$ has hair so-and-so' (for sake of simplicity we suppose that there are no possible bald US presidents in 2017); it follows that two possible US presidents in 2017 stand in the suitable relation of resemblance iff they are indistinguishable with respect to their hairs; and if two possible US presidents in 2017 are indistinguishable with respect to their hairs, then it is impossible that one is blond while the other is not.

We may define the following criterion based in a pattern of resemblance as previously defined:

\section{Good recarvings (GR)}


Let $S$ be a sentence exactly about $m$, and let $U_{S}$ be the set of witnesses of $S$. Let $\Sigma$ be an operation of subject matter restriction, such that $\Sigma(m)=n$ with $n$ strictly included in $m$. We say that $\Sigma$ is a good candidate for content recarving iff there is an intrinsic determinable property $P$ shared by all witnesses in $U_{S}$ whose class of intrinsic determinates $\Delta\left(U_{S}\right)$ satisfies the following:

i. $\quad$ For every two possible worlds $w_{1}, w_{2}$ and for every $X$ in $\Delta\left(U_{S}\right)$, both $m\left(w_{1}\right)$ and $m\left(w_{2}\right)$ have $X$ iff $w_{1}$ and $w_{2}$ belong to the same $n$-cell,

ii. For every two possible worlds $w_{1}, w_{2}$ and for every $X$ in $\Delta\left(U_{S}\right)$, if $m\left(w_{1}\right)$ and $m\left(w_{2}\right)$ have $X$, then the truth-value of $S$ in $w_{1}$ is identical to the truth-value of $S$ in $w_{2}$.

The criterion (GR) may be seen as suggesting how to define the new restricted subject matter by merging the cells of the initial subject matter once that a range of determinate properties of a certain determinable is given. Indeed, according to condition (i), whenever two witnesses of $S$ have the same determinate, we must merge their corresponding $m$-cells. Condition (ii) ensures the relevance of the pattern of resemblances: two witnesses that have the same determinate must be either both ways of being true of $S$ or both ways of being false of $S$. Note the difference between (CESM) and (GR): while the former is framed in terms of the relation of determination between properties of possible worlds, the latter is framed in terms of the relation of determination between properties of witnesses.

How (GR) is supposed to grant that the resulting subject matter $n$ is an effective one? The answer is almost immediate: to say that two witnesses of $S$ have the same determinate is just to say that they are indistinguishable under a fixed respect; as a consequence, all worlds in which 
these witnesses exist are also indistinguishable under the same fixed respect. In other words, the relation of determination between intrinsic properties of the witnesses of a given sentence is transmitted between corresponding properties of possible worlds, and - by (CESM) - the new subject matter is effective.

To use the relation of determination betw een properties to define a good candidate operation for content recarving appears to be an illuminating improvement on the definition of the previous section: content recarving is not just a subject matter restriction, yet a restriction that has been characterized as a relevant specification; of all subject matters strictly included in the initial one, the operation of content recarving should pick out only those that represent a further determination of what the given sentence says about its subject matter. For this reason, we cannot perform a recarving of the sentence 'The US president in 2017 is blond' by restricting the subject matter to ${ }^{\circ}$ The left hand of the US president in 2017': for there are possible US presidents in 2017 that are indistinguishable with respect to their left hands while being distinguishable with respect to their blondness; what the sentence says about the US president in 2017 has nothing to do with the his/her left hand. Nor we may perform the recarving by an arbitrary subpartitioning of the initial subject matter, for what grants that worlds belonging to the same new cell really are indistinguishable under a certain respect?

What such an improved account of content recarving has to say regarding abstraction principles? Consider the case of Hume's Principle: 'The number of stars $=$ the number of planets' is expected to be the recarving of 'There are as many stars as planets'. The latter sentence is exactly about the concept of star, the concept of planet. Each cell in which this subject matter partitions the logical space, corresponds to worlds agreeing on all instances of the concept of star and that of planet. Consider the property "The concept $X$ is 
$n$-instantiated': for every finite cardinal $n$, it is well known that such a property may be defined without mentioning cardinal numbers, but relying only on a suitable use of quantifiers of the form $\exists_{n} x X x$. This second-order property may be seen as an intrinsic determinate of the second-order determinable ' $X$ is instantiated'. The list of all second-order properties $\neg \exists x X x, \exists_{1} x X x, \exists_{2} x X x, \ldots$, $\exists_{n} \times X x, \ldots$, may be considered as the class of determinates defining our pattern of resemblances according to the criterion (GR). Call this class $C_{\# ;}$; we will define the relation

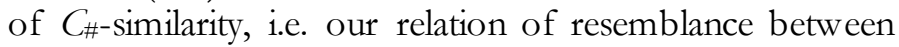
two pairs of extension of respectively the concept of star and the concept of planet: for any two possible worlds $w_{1}$, $w_{2}$, the pair of extensions of the concept of star and the

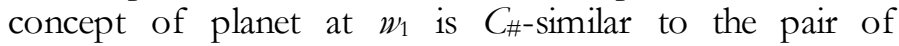
extensions of the concept of star and the concept of planet at $w_{2}$ iff the extensions of the concept of star at $w_{1}$ and $w_{2}$ have the same determinate in $C_{\#}$ and the same happens with the pair of extensions of the concept of planet.

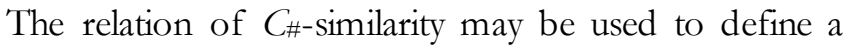
new equivalence relation $\equiv_{\#}$ on possible worlds: for any two worlds $w_{1}, w_{2} \in \mathbf{W}, w_{1} \equiv_{\#} w_{2}$ iff the pair composed by the extension of the concept of star in $w_{1}$ and extension of the concept of planet in $w_{1}$ is $C_{\#-}$ similar to the pair composed by the extension of the concept of star in $w_{2}$ and the extension of the concept of planet in $w_{2}$. The relation $\equiv_{\#}$ defines the new subject matter strictly included in the concept of star, the concept of planet. It should appear clear what this new subject matter is:

〈\#( the concept of star), \#(the concept of planet) 〉

where the symbol \# has been used to abbreviate the expression 'the cardinal number of'. This result shows that it is possible - by an application of the recarving operation according to a certain pattern of resemblances - to restrict the subject matter of a sentence saying that two concepts 
are equinumerous so that a new subject matter, the cardinal numbers of the two concepts at issue, is determined. Moreover, by (GR), the new subject matter is an effective one, for it is defined according to a relation of determination between higher-order properties. What is still missing is a way of deciding whether there is a sentence exactly about the restricted subject matter or not. In other words, what is needed is a criterion for saying that the new cells in which the logical space has been partitioned may be associated with ways of being true (false) of a certain sentence, i.e. with existing entities. This takes us to the second problem related to the definition of a non-arbitrary recarving operation. The discussion that follows, though being focused on Hume's Principle, is applicable to all cases of abstraction principles.

The given definition of content recarving and the described procedure of the determination of the restricted subject matter according to a pattern of resemblance, allow us to classify the possible scenarios in three cases:

1. Even if the subject matter cardinal number may be determined according to the described procedure, there is no sentence exactly about it, i.e. the cells determined by the subject matter cardinal number cannot be associated with ways of being true (false) of a certain sentence, simply because the objects that should play the role of witnesses of such a sentence (i.e. the cardinal numbers) do not exist in all possible worlds. Verdict: cardinal number is an effective subject matter, it is not an exact subject matter of a certain sentence, and there are numberless worlds.

2. Cardinal number is a subject matter and the sentence 'The number of stars $=$ the number of planets' is exactly about cardinal numbers. Yet a difference in the way this sentence is true (false) may 
be explained without appealing to cardinal numbers as objects existing in every possible worlds. Verdict: cardinal number is an effective subject matter, it corresponds to the exact subject matter of a certain class of sentences, yet there are numberless worlds.

3. Cardinal number is a subject matter and the sentence ${ }^{\circ}$ The number of stars $=$ the number of planets' is exactly about cardinal numbers. The way this sentence is true or false is characterized in terms of the objects corresponding to the cardinal number of stars and that of planets; in other words, cardinal numbers as objects are the witnesses of the sentence 'The number of stars $=$ the number of planets'. Verdict: cardinal number is an effective subject matter, it corresponds to the exact subject matter of a certain class of sentences, and there are cardinal numbers in every possible world.

All cases agree on the fact that cardinal number is an effective subject matter: sentences may be about cardinal numbers in a genuine sense. For instance, the sentence 'There are as many stars as planets' is about both the number of stars and the number of planets, for this latter subject matter is effective and included into the exact subject matter of the sentence. Clearly, 'There are as many stars as planets' is not exactly about cardinal numbers: for cardinal numbers are not crucial for the determination of the ways in which the sentence may be true or false. Case 1 is the only case of rejection of the fact that 'The number of stars $=$ the number of planets' is the result of recarving the content of 'There are as many stars as planets': for the former sentence cannot be exactly about the restricted subject matter determined by the described procedure. Case 2 and 3 present an agreement on the fact that the sentence of number identity is a recarving of the sentence of 
equinumerousity between two concepts. Case 3 accepts the recarving in the most straightforward way: a sentence exactly about cardinal numbers is made true or false by the objects corresponding to the cardinal numbers at issue. Case 2 is more complicated, yet far more interesting: it contemplates the possibility of formulating sentences exactly about cardinal numbers which do not require the necessary existence of them as objects. How this could be possible? How the notion of exact subject matter may be characterized without the relation between ways of being true/false and existing verifiers/falsifiers? A preliminary answer could be that the ways in which the sentence 'The number of stars $=$ the number of planets' is true may be characterized pragmatically; for instance, we may agree on a conventional procedure-type of cardinal number assignment, e.g. counting, and we may establish the validity of such procedures even in possible worlds in which there are no beings able to count. Another possible answer uses the notion of the best world when a certain subject matter is concerned. The sentence 'The number of stars $=$ the number of planets' is true (false) in a certain possible world $w$ precisely in the way in which it is true (false) in all worlds belonging to the same cardinal number-partition that are most adequate to the subject matter cardinal number: and these worlds are all worlds in which cardinal numbers exist ${ }^{16}$.

There is much more to say about the ontological import of the operation of content recarving; yet disputing the details of this issue will take us too faraway. What matters for the present discussion is the fact that the recarving operation may be characterized as a restriction of subject matter where the restriction is intended as a specification relevant to what the sentence to be recarved says about its exact subject matter.

16 Both the pragmatic answer and the best world answer are suggested by Yablo (2014), p.36

Manuscrito-Rev. Int. Fil. Campinas, v. 42, n. 1, pp. 45-90, Jan-Mar. 2019. 


\section{CONCLUSION}

I have proposed that the operation of carving up a content expressed by a sentence $\varphi$ may be conceived as the partitioning of the bilateral proposition expressed by $\varphi$ according to the exact subject matter of $\varphi$; moreover, I have proposed that the recarving of a content already carved up by a sentence $\varphi$ may be seen as anoperation that restricts the given subject matter in accordance with what $\varphi$ says about its subject matter. Such a notion of restriction in accordance with what $\varphi$ says about its subject matter has been characterized in terms of the relation of determination between properties; the resulting account proposes content recarving as an operation of subject matter restriction, where restriction is intended as a relevant specification.

As I hope, this proposal clarifies the apparently mysterious procedure described in \$64 of the Foundations of Arithmetic the application of the specific content of the symbol 'll' to $a$ and $b$ is interpreted as the restriction of the subject matter (i.e. line $a$, line $b$ ) to the aspect most relevant to what ' $a \| b$ ' says about it: directions. As a result of this restriction of subject matter, the new condition applied to the new subject matter has to be more general, and in this respect I also hope to have clarified what is the result of "removing what is specific in the content of ".

The proposal has the advantage of explaining the operation of content recarving using a philosophically significant notion - that of subject matter - with reference to a robust theoretical characterization of this notion (Lewis-Yablo theory of subject matter).

The fact that the proposal presents several limitations insofar as it is neither a proper interpretation of Frege's view nor a characterization within Frege's conceptual universe, has the advantage of resulting more general in its applications: indeed, the described recarving operation is not limited to the case of abstraction principles. 
There are two main possible future works stemming from the given characterization. The first one is the analysis of other abstraction principles: the outcome of such an analysis is that some abstraction principles may not be considered as obtained by content recarving, for they may not perform an effective subject matter restriction. The second one concerns the relation between mathematical language and subject matter: arithmetical statements, when conceived as obtained by a recarving operation, may be seen as sentences that while succeeding in being about mathematical notions, are not committed to the existence of mathematical objects.

This reasearch was funded by the FAPESP

grant n. 2014/27057-5

\section{REFERENCES}

Armstrong, D. M. A World of States of Affairs. Cambridge University Press, 1996.

Dummett, M. Frege, Philosophy of Mathematics. Duckwell, 1991.

FREGE, G. Foundations of Arithmetic. Routledge, 1950.

Frege, G., Geach, P. T., and Black, M. On Concept and Object. Mind, 60(238):168-180, 1951.

Goodman, N. About. Mind, 70:1, 1961.

Hale, B. Grundlagen \64. Proceedings of the Aristotelian Society, 97(3):243- 261, 1997.

Johnson, W. E. Logic, Part 1. Cambridge University Press, 2014.

Manuscrito-Rev. Int. Fil. Campinas, v. 42, n. 1, pp. 45-90, Jan-Mar. 2019. 
Körner, S. and SEarle, J. On Determinables and Resemblance. Aristotelian Society Supplementary Volume, 33(1):125-158, 1959.

LEWIS, D. Statements Partly About Observation Philosophical Papers, 17(1):1-31, 1988.

PotTer, M. and Smiley, T. Abstraction by Recarving. Proceedings of the Aristotelian Society, 101(3):327-338, 2001.

PrIOR, A. N. Determinables, Determinates and Determinants (ii). Mind, 58(230):178-194, 1949.

RicketTs, T. and PotTer, M. The Cambridge Companion to Frege. Cambridge University Press, 2010.

RYLE, G. 'About'. Analysis, 1(1):10-12, 1933.

YABLO, S. Mental Causation. Philosophical Review, 101(2):245-280, 1992.

Carving the Content at its Joints. Canadian Journal of Philosophy, 38. pp.145-177, 2008. Aboutness. Princeton University Press, 2014.

\section{(cc) $\mathbf{E Y}$}

